\title{
THE KITAI CRITERION AND BACKWARD SHIFTS
}

\author{
STANISLAV SHKARIN
}

(Communicated by Jonathan M. Borwein)

\begin{abstract}
It is proved that for any separable infinite dimensional Banach space $X$, there is a bounded linear operator $T$ on $X$ such that $T$ satisfies the Kitai criterion. The proof is based on a quasisimilarity argument and on showing that $I+T$ satisfies the Kitai criterion for certain backward weighted shifts $T$.
\end{abstract}

\section{INTRODUCTION}

All vector spaces are assumed to be over $\mathbb{K}$ being either the field $\mathbb{C}$ of complex numbers or the field $\mathbb{R}$ of real numbers. As usual, $\mathbb{Z}$ is the set of integers, $\mathbb{Z}_{+}$is the set of non-negative integers and $\mathbb{N}$ is the set of positive integers. For a Banach space $X$, the symbol $L(X)$ stands for the space of bounded linear operators on $X$ and $X^{*}$ is the space of continuous linear functionals on $X$.

Definition 1. Let $X$ be a Banach space and $T \in L(X)$. We say that $T$ is hypercyclic if there exists $x \in X$ such that the orbit $\left\{T^{n} x: n \in \mathbb{Z}_{+}\right\}$is dense in $X$. Following Ansari [1], $T$ is called hereditarily hypercyclic if for any infinite set $\Lambda \subseteq \mathbb{Z}_{+}$, there exists $x \in X$ for which $\left\{T^{n} x: n \in \Lambda\right\}$ is dense in $X$.

Remark. Hypercyclic operators have been intensely studied during the last few decades; see surveys $[14,15]$ and the references therein. It is also worth noting that in the terminology of $[8,13]$ hereditarily hypercyclic operators are called hereditarily hypercyclic with respect to the sequence $n_{k}=k$ of all non-negative integers.

Definition 2. We say that a bounded linear operator $T$ on a Banach space $X$ satisfies the Kitai criterion [16] if there exist two dense subsets $E$ and $F$ of $X$ and a map $S: F \rightarrow F$ such that $T S y=y, S^{k} y \rightarrow 0$ and $T^{k} x \rightarrow 0$ as $k \rightarrow \infty$ for any $y \in F$ and $x \in E$.

Definition 3. Let $X$ be a topological space. A continuous map $T: X \rightarrow X$ is called mixing if for any two non-empty open sets $U, V \subseteq X, T^{n}(U) \cap V \neq \varnothing$ for all sufficiently large $n \in \mathbb{N}$.

Received by the editors November 9, 2006.

2000 Mathematics Subject Classification. Primary 47A16, 37A25.

Key words and phrases. Hypercyclic operators, mixing operators, the Kitai criterion, biorthogonal sequences, backward shifts, quasisimilarity.

The author was partially supported by Plan Nacional I+D+I grant no. MTM2006-09060, Junta de Andalucía FQM-260 and British Engineering and Physical Research Council Grant GR/T25552/01. 
It is well known that a bounded linear operator $T$ on a Banach space is mixing if and only if it is hereditarily hypercyclic; see [13]. Moreover, if $T$ satisfies the Kitai criterion, then $T$ is mixing; see [13, 10]. Grivaux [13], answering a question raised by Shapiro, proved that for any separable infinite dimensional Banach space $X$, there exists a mixing $T \in L(X)$ that does not satisfy the Kitai criterion. We address the question raised by Petersson (Miniworkshop: Hypercyclicity and Linear Chaos, Oberwolfach, August, 2006) whether there is a bounded linear operator satisfying the Kitai criterion on any separable infinite dimensional Banach space. The following theorem gives an affirmative answer to this question.

Theorem 1.1. Let $X$ be a separable infinite dimensional Banach space. Then there exists $T \in L(X)$ satisfying the Kitai criterion.

This theorem fits into the following chain of results. Herzog [12] proved that there is a supercyclic continuous linear operator on any separable infinite dimensional Banach space. Later Ansari [2] and Bernal-González [7] showed independently that for any separable infinite dimensional Banach space $X$ there is a hypercyclic operator $T \in L(X)$. Finally, as we have already mentioned, Grivaux [13] proved that there is a mixing operator on any separable infinite dimensional Banach space.

Recall that a backward weighted shift on $\ell_{p}=\ell_{p}\left(\mathbb{Z}_{+}\right)$for $1 \leqslant p<\infty$ or $c_{0}=$ $c_{0}\left(\mathbb{Z}_{+}\right)$is the operator $T$ acting on the canonical basis $\left\{e_{n}\right\}_{n=0}^{\infty}$ as follows: $T e_{0}=0$ and $T e_{n}=w_{n} e_{n-1}$ for $n \geqslant 1$, where $w=\left\{w_{n}\right\}_{n \in \mathbb{N}}$ is a bounded sequence of nonzero numbers in $\mathbb{K}$. The proof of Theorem 1.1 is based on the fact that operators $I+T$ satisfy the Kitai criterion for certain backward weighted shifts $T$. Namely, the following theorem holds.

Theorem 1.2. Let $X$ be either $\ell_{p}$ with $1 \leqslant p<\infty$ or $X=c_{0}$ and let $T: X \rightarrow X$ be a backward weighted shift with the weight sequence $\left\{w_{n}\right\}_{n \in \mathbb{N}}$. Assume also that

$$
\varliminf_{n \rightarrow \infty}\left(n ! \prod_{j=1}^{n}\left|w_{j}\right|\right)^{1 / n}>0 .
$$

Then $I+T$ satisfies the Kitai criterion.

It is worth noting that if there exists $c>0$ such that $\left|w_{n}\right| \geqslant c / n$ for each $n \in \mathbb{N}$, then (1.1) is satisfied. Thus, we have the following corollary.

Corollary 1.3. Let $X$ be either $\ell_{p}$ with $1 \leqslant p<\infty$ or $X=c_{0}$ and let $T: X \rightarrow X$ be a backward weighted shift with the weight sequence $\left\{w_{n}\right\}_{n \in \mathbb{N}}$. Assume also that there exists $c>0$ such that $\left|w_{n}\right| \geqslant c / n$ for each $n \in \mathbb{N}$. Then $I+T$ satisfies the Kitai criterion.

Recall that according to a theorem by Salas [19] the operator $I+T$ is hypercyclic for any backward weighted shift $T$ on $\ell_{p}$ with $1 \leqslant p<\infty$. Grivaux [13] has observed that basically the same proof allows us to show that the operators $I+T$ in the Salas theorem are mixing. Theorem 1.2 shows that under certain conditions on the weight sequence these operators satisfy the Kitai criterion.

Theorems 1.1 and 1.2 are proved in Section 2. Section 3 is devoted to concluding remarks.

\section{Operators SATISFYing the KitAi CRITERION}

In this section we shall prove Theorems 1.1 and 1.2. We need some preparation. 
2.1. Biorthogonal sequences. We are going to prove the existence of biorthogonal sequences with certain properties on any separable infinite dimensional Banach space. Recall that a family $\left\{\left(x_{\alpha}, f_{\alpha}\right): \alpha \in A\right\}$, where the $x_{\alpha}$ are vectors in a Banach space $X$ and $f_{\alpha} \in X^{*}$, is called biorthogonal if $f_{\alpha}\left(x_{\beta}\right)=\delta_{\alpha, \beta}$ for any $\alpha, \beta \in A, \delta_{\alpha, \beta}$ being the Kronecker delta, that is, $\delta_{\alpha, \beta}=1$ if $\alpha=\beta$ and $\delta_{\alpha, \beta}=0$ if $\alpha \neq \beta$.

Proposition 2.1. Let $X$ be a separable infinite dimensional Banach space, let $\left\{b_{k}\right\}_{k \in \mathbb{Z}_{+}}$be a sequence of numbers in $[3, \infty)$ such that $b_{k} \rightarrow \infty$ as $k \rightarrow \infty$ and let $\left\{n_{k}\right\}_{k \in \mathbb{Z}_{+}}$be a strictly increasing sequence of positive integers such that $n_{0}=0$ and $n_{k+1}-n_{k} \geqslant 2$ for each $k \in \mathbb{Z}_{+}$. Then there exists a biorthogonal sequence $\left\{\left(x_{k}, f_{k}\right)\right\}_{k \in \mathbb{Z}_{+}}$in $X \times X^{*}$ such that

(B1) $\left\|x_{k}\right\|=1$ for each $k \in \mathbb{Z}_{+}$;

(B2) $\operatorname{span}\left\{x_{k}: k \in \mathbb{Z}_{+}\right\}$is dense in $X$;

(B3) $\left\|f_{n_{k}}\right\| \leqslant b_{k}$ for each $k \in \mathbb{Z}_{+}$;

(B4) $\left\|f_{j}\right\| \leqslant 3$ if $j \in \mathbb{Z}_{+} \backslash\left\{n_{k}: k \in \mathbb{Z}_{+}\right\}$;

(B5) for any $k \in \mathbb{Z}_{+}$and any numbers $c_{j} \in \mathbb{K}$ with $n_{k}+1 \leqslant j \leqslant n_{k+1}-1$,

$$
\frac{1}{2}\left\|\sum_{j=n_{k}+1}^{n_{k+1}-1} c_{j} x_{j}\right\| \leqslant\left(\sum_{j=n_{k}+1}^{n_{k+1}-1}\left|c_{j}\right|^{2}\right)^{1 / 2} \leqslant 2\left\|\sum_{j=n_{k}+1}^{n_{k+1}-1} c_{j} x_{j}\right\| .
$$

The proof of the above proposition is based on the following theorem, which is known as the Dvoretzky theorem on almost spherical sections [11].

Theorem D. For each $n \in \mathbb{N}$ and each $\varepsilon>0$, there exists $m=m(n, \varepsilon) \in \mathbb{N}$ such that for any Banach space $X$ with $\operatorname{dim} X \geqslant m$ there is an $n$-dimensional linear subspace $L$ in $X$ and a linear basis $e_{1}, \ldots, e_{n}$ in $L$ for which $\left\|e_{j}\right\|=1$ for $1 \leqslant j \leqslant n$ and

$$
\frac{1}{1+\varepsilon}\left\|\sum_{j=1}^{n} c_{j} e_{j}\right\| \leqslant\left(\sum_{j=1}^{n}\left|c_{j}\right|^{2}\right)^{1 / 2} \leqslant(1+\varepsilon)\left\|\sum_{j=1}^{n} c_{j} e_{j}\right\| \quad \text { for any }\left(c_{1}, \ldots, c_{n}\right) \in \mathbb{K}^{n} .
$$

We also need the following technical lemmas.

Lemma 2.2. Let $A$ be an infinite countable set, let $\left\{a_{n}\right\}_{n \in A}$ be a sequence of nonnegative numbers such that there exists $c>0$ for which the set $\left\{n \in A: a_{n} \leqslant c\right\}$ is infinite and let $\left\{b_{n}\right\}_{n \in \mathbb{Z}_{+}}$be a sequence of numbers in $[c, \infty)$ such that $b_{n} \rightarrow \infty$ as $n \rightarrow \infty$. Then there exists a bijection $\pi: \mathbb{Z}_{+} \rightarrow A$ such that $a_{\pi(n)} \leqslant b_{n}$ for each $n \in \mathbb{Z}_{+}$.

Proof. Since the set $\left\{n \in A: a_{n} \leqslant c\right\}$ is infinite, we can choose two disjoint infinite subsets $B$ and $C$ of $A$ such that $a_{n} \leqslant c$ for each $n \in B$ and $B \cup C=A$. Fix a bijection $\varphi: \mathbb{Z}_{+} \rightarrow C$. Since $b_{n} \rightarrow \infty$ as $n \rightarrow \infty$, we can choose a sequence $\left\{j_{k}\right\}_{k \in \mathbb{Z}_{+}}$of non-negative integers such that $j_{k+1}-j_{k} \geqslant 2$ and $a_{\varphi(k)} \leqslant b_{j_{k}}$ for each $k \in \mathbb{Z}_{+}$. Denote $D=\mathbb{Z}_{+} \backslash\left\{j_{k}: k \in \mathbb{Z}_{+}\right\}$. Since $j_{k+1}-j_{k} \geqslant 2$, the set $D$ is infinite. Since both $B$ and $D$ are infinite and countable, there exists a bijection $\psi: D \rightarrow B$. Now consider the map $\pi: \mathbb{Z}_{+} \rightarrow A$ defined by the formula

$$
\pi(n)= \begin{cases}\varphi(k) & \text { if } n=j_{k} \\ \psi(n) & \text { if } n \in D\end{cases}
$$

Clearly $\pi$ is a bijection. If $n \in D$, we have $a_{\pi(n)}=a_{\psi(n)} \leqslant c \leqslant b_{n}$. Indeed, $a_{m} \leqslant c$ for $m \in B$ and $c \leqslant b_{n}$ for $n \in \mathbb{Z}_{+}$. If $n=j_{k}$, we have $a_{\pi(n)}=a_{\varphi(k)} \leqslant b_{j_{k}}=b_{n}$. Thus, $a_{\pi(n)} \leqslant b_{n}$ for each $n \in \mathbb{Z}_{+}$. 
Lemma 2.3. Let $X$ be an infinite dimensional Banach space, let $u \in X$ and let $\left\{\left(x_{k}, f_{k}\right): 1 \leqslant k \leqslant n\right\}$ be a finite biorthogonal sequence in $X \times X^{*}$. Then there exist $x_{n+1} \in X$ and $f_{n+1} \in X^{*}$ such that $\left\|x_{n+1}\right\|=1,\left\{\left(x_{k}, f_{k}\right): 1 \leqslant k \leqslant n+1\right\}$ is biorthogonal and $u \in \operatorname{span}\left\{x_{1}, \ldots, x_{n+1}\right\}$.

Proof. Since $X=L \oplus N$, where $L=\operatorname{span}\left\{x_{1}, \ldots, x_{n}\right\}$ and $N=\bigcap_{k=1}^{n} \operatorname{ker} f_{j}$, we can pick a vector $x_{n+1} \in N$ such that $\left\|x_{n+1}\right\|=1$ and $u \in M=$ $\operatorname{span}\left(L \cup\left\{x_{n+1}\right\}\right)=\operatorname{span}\left\{x_{1}, \ldots, x_{n+1}\right\}$. Since $x_{1}, \ldots, x_{n+1}$ is a linear basis in $M$, there exists a unique linear functional $g: M \rightarrow \mathbb{K}$ such that $g\left(x_{j}\right)=0$ for $1 \leqslant j \leqslant n$ and $g\left(x_{n+1}\right)=1$. According to the Hahn-Banach theorem, there exists $f_{n+1} \in X^{*}$ such that $\left.f_{n+1}\right|_{M}=g$. Clearly the pair $\left(x_{n+1}, f_{n+1}\right)$ satisfies all the required conditions.

Lemma 2.4. Let $m \in \mathbb{N}, X$ be an infinite dimensional Banach space and let $\left\{\left(x_{k}, f_{k}\right): 1 \leqslant k \leqslant n\right\}$ be a finite biorthogonal sequence in $X \times X^{*}$. Then there exist $x_{n+1}, \ldots, x_{n+m} \in X$ and $f_{n+1}, \ldots, f_{n+m} \in X^{*}$ such that $\left\{\left(x_{k}, f_{k}\right): 1 \leqslant k \leqslant n+m\right\}$ is biorthogonal, $\left\|x_{k}\right\|=1$ and $\left\|f_{k}\right\| \leqslant 3$ for $n+1 \leqslant k \leqslant n+m$ and

$\frac{1}{2}\left\|\sum_{j=n+1}^{n+m} c_{j} x_{j}\right\| \leqslant\left(\sum_{j=n+1}^{n+m}\left|c_{j}\right|^{2}\right)^{1 / 2} \leqslant 2\left\|\sum_{j=n+1}^{n+m} c_{j} x_{j}\right\|$ for any $\left(c_{n+1}, \ldots, c_{n+m}\right) \in \mathbb{K}^{m}$.

Proof. Fix $\delta \in(0,1 / 2)$ such that $\frac{(1+\delta)(2-\delta)}{1-\delta}<3$ and let $L=\operatorname{span}\left\{x_{1}, \ldots, x_{n}\right\}$, $S=\{x \in L:\|x\|=1\}$. Since $L$ is finite dimensional, $S$ is a compact metric space with respect to the metric inherited from $X$. Hence we can find a finite set $\left\{u_{1}, \ldots, u_{r}\right\} \subset S$ such that for each $x \in S$ there exists $j \in\{1, \ldots, r\}$ for which $\left\|x-u_{j}\right\|<\delta$. According to the Hahn-Banach theorem, there exist functionals $\varphi_{1}, \ldots, \varphi_{r} \in X^{*}$ such that $\left\|\varphi_{j}\right\|=\varphi_{j}\left(u_{j}\right)=1$ for $1 \leqslant j \leqslant r$. Consider the space

$$
M=\bigcap_{l=1}^{n} \operatorname{ker} f_{j} \cap \bigcap_{j=1}^{r} \operatorname{ker} \varphi_{j} .
$$

Since $X$ is infinite dimensional, $M$ is a closed infinite dimensional subspace of $X$. By Theorem D there exist $x_{n+1}, \ldots, x_{n+m} \in M$ such that $\left\|x_{j}\right\|=1$ for $n+1 \leqslant j \leqslant$ $n+m$ and

$$
\begin{aligned}
\frac{1}{1+\delta}\left\|\sum_{j=n+1}^{n+m} c_{j} x_{j}\right\| & \leqslant\left(\sum_{j=n+1}^{n+m}\left|c_{j}\right|^{2}\right)^{1 / 2} \\
& \leqslant(1+\delta)\left\|\sum_{j=n+1}^{n+m} c_{j} x_{j}\right\| \text { for any }\left(c_{n+1}, \ldots, c_{n+m}\right) \in \mathbb{K}^{m} .
\end{aligned}
$$

Since $\delta \leqslant 1 / 2,(2.2)$ implies $(2.1)$.

Now let $L_{0}=\operatorname{span}\left\{x_{n+1}, \ldots, x_{n+m}\right\}$. Since $L_{0} \subset M$ and the linear functionals $f_{j}$ for $1 \leqslant j \leqslant n$ vanish on $M$, we see that the vectors $x_{1}, \ldots, x_{n+m}$ form a linear basis in $L \oplus L_{0}$. Thus, there exist unique linear functionals $g_{n+1}, \ldots, g_{n+m}$ on $L \oplus L_{0}$ such that $g_{j}\left(x_{k}\right)=\delta_{j, k}$ for $n+1 \leqslant j \leqslant n+m$ and $1 \leqslant k \leqslant n+m$. We shall estimate the norms of $g_{j}$.

First, let $y=\sum_{j=n+1}^{n+m} c_{j} x_{j} \in L_{0}$ and $n+1 \leqslant k \leqslant n+m$. Using (2.2), we obtain

$$
\left|g_{k}(y)\right|=\left|c_{k}\right| \leqslant\left(\sum_{j=n+1}^{n+m}\left|c_{j}\right|^{2}\right)^{1 / 2} \leqslant(1+\delta)\|y\| .
$$


Thus,

$$
\left|g_{k}(y)\right| \leqslant(1+\delta)\|y\| \quad \text { for each } y \in L_{0} .
$$

Now let $x \in L$ with $\|x\|=1$ and $y \in L_{0}$. Pick $j \in\{1, \ldots, r\}$ such that $\left\|x-u_{j}\right\| \leqslant \delta$.

Then

$$
\|x+y\| \geqslant\left|\varphi_{j}(x+y)\right|=\left|\varphi_{j}(x)\right| \geqslant\left|\varphi_{j}\left(u_{j}\right)\right|-\left|\varphi_{j}\left(x-u_{j}\right)\right| \geqslant 1-\delta .
$$

It follows that $\|x+y\| \geqslant(1-\delta)\|x\|$ for each $x \in L$ and $y \in L_{0}$. Consequently

$$
\begin{aligned}
\|y\| \leqslant\|x\|+\|x+y\| \leqslant\left(1+\frac{1}{1-\delta}\right)\|x+y\|= & \frac{2-\delta}{1-\delta}\|x+y\| \\
& \text { for each } x \in L \text { and } y \in L_{0} .
\end{aligned}
$$

Now let $w \in L \oplus L_{0}$. Then there exist a unique $x \in L$ and a $y \in L_{0}$ for which $w=x+y$. Applying (2.3) and (2.4), we obtain

$$
\left|g_{k}(w)\right|=\left|g_{k}(x+y)\right|=\left|g_{k}(y)\right| \leqslant(1+\delta)\|y\| \leqslant \frac{(1+\delta)(2-\delta)}{1-\delta}\|w\| .
$$

That is, $\left\|g_{k}\right\| \leqslant \frac{(1+\delta)(2-\delta)}{1-\delta} \leqslant 3$ for $n+1 \leqslant k \leqslant n+m$. Using the Hahn-Banach theorem, we can now choose $f_{k} \in X^{*}$ for $n+1 \leqslant k \leqslant n+m$ such that $\left.f_{k}\right|_{L \oplus L_{0}}=g_{k}$ and $\left\|f_{k}\right\| \leqslant 3$. It remains to observe that $\left\{\left(x_{k}, f_{k}\right): 1 \leqslant k \leqslant n+m\right\}$ is a biorthogonal sequence satisfying all desired conditions.

Proof of Proposition 2.1. Since $X$ is separable, we can choose a sequence $\left\{u_{k}\right\}_{k \in \mathbb{Z}_{+}}$ of vectors in $X$ such that $\operatorname{span}\left\{u_{k}: k \in \mathbb{Z}_{+}\right\}$is dense in $X$. Denote $m_{k}=n_{k}+k$ for $k \in \mathbb{Z}_{+}$. First, according to the Hahn-Banach theorem, we can pick $\left(y_{0}, g_{0}\right) \in$ $X \times X^{*}$ such that $\left\|y_{0}\right\|=\left\|g_{0}\right\|=1$ and $u_{0} \in \operatorname{span}\left\{y_{0}\right\}$, which will serve as the basis of an inductive process. Now we shall show inductively that for $k=0,1, \ldots$, there exist $\left\{\left(y_{j}, g_{j}\right): m_{k}<j \leqslant m_{k+1}\right\} \subset X \times X^{*}$ such that

(P1) $\left\{\left(y_{j}, g_{j}\right): 0 \leqslant j \leqslant m_{k+1}\right\}$ is a biorthogonal sequence;

(P2) $\left\|y_{j}\right\|=1$ for $m_{k}<j \leqslant m_{k+1}$;

(P3) $u_{k+1} \in \operatorname{span}\left\{y_{0}, \ldots, y_{m_{k+1}}\right\}$;

(P4) $\left\|g_{j}\right\| \leqslant 3$ for $m_{k}<j<m_{k+1}$;

(P5) for any $\left(c_{m_{k}+1}, \ldots, c_{m_{k+1}-1}\right) \in \mathbb{K}^{m_{k+1}-m_{k}-1}$,

$$
\frac{1}{2}\left\|\sum_{j=m_{k}+1}^{m_{k+1}-1} c_{j} y_{j}\right\| \leqslant\left(\sum_{j=m_{k}+1}^{m_{k+1}-1}\left|c_{j}\right|^{2}\right)^{1 / 2} \leqslant 2\left\|\sum_{j=m_{k}+1}^{m_{k+1}-1} c_{j} y_{j}\right\| .
$$

Suppose $\left(y_{j}, g_{j}\right)$ for $j \leqslant m_{k}$ satisfying the desired properties are already constructed. Using Lemma 2.4 , we find $\left(y_{j}, g_{j}\right)$ for $m_{k}<j<m_{k+1}$ in $X \times X^{*}$ such that $\left\{\left(y_{j}, g_{j}\right): 0 \leqslant j<m_{k+1}\right\}$ is a biorthogonal sequence, $\left\|y_{j}\right\|=1$ for $m_{k}<j<m_{k+1}$ and the conditions (P4) and (P5) are satisfied. By Lemma 2.3, we can now choose $\left(y_{m_{k+1}}, g_{m_{k+1}}\right) \in X \times X^{*}$ such that $\left\|y_{m_{k+1}}\right\|=1$ and the conditions (P1) and (P3) are satisfied. Therefore $\left\{\left(y_{j}, g_{j}\right): m_{k}<j \leqslant m_{k+1}\right\}$ satisfy (P1)-(P5).

Thus we have just defined an inductive procedure, which provides a sequence $\left\{\left(y_{j}, g_{j}\right): j \in \mathbb{Z}_{+}\right\}$of elements of $X \times X^{*}$ satisfying

(Q1) $\left\{\left(y_{j}, g_{j}\right): j \in \mathbb{Z}_{+}\right\}$is a biorthogonal sequence;

(Q2) $\left\|y_{j}\right\|=1$ for $j \in \mathbb{Z}_{+}$;

(Q3) $\operatorname{span}\left\{u_{j}: j \in \mathbb{Z}_{+}\right\} \subseteq \operatorname{span}\left\{y_{j}: j \in \mathbb{Z}_{+}\right\}$;

(Q4) $\left\|g_{j}\right\| \leqslant 3$ for $m_{k}<j<m_{k+1}, k \in \mathbb{Z}_{+}$; 
(Q5) for any $k \in \mathbb{Z}_{+}$and $\left(c_{m_{k}+1}, \ldots, c_{m_{k+1}-1}\right) \in \mathbb{K}^{m_{k+1}-m_{k}-1}$,

$$
\frac{1}{2}\left\|\sum_{j=m_{k}+1}^{m_{k+1}-1} c_{j} y_{j}\right\| \leqslant\left(\sum_{j=m_{k}+1}^{m_{k+1}-1}\left|c_{j}\right|^{2}\right)^{1 / 2} \leqslant 2\left\|\sum_{j=m_{k}+1}^{m_{k+1}-1} c_{j} y_{j}\right\| .
$$

Now we shall show that a biorthogonal sequence with the desired properties can be obtained as a permutation of the biorthogonal sequence $\left\{\left(y_{j}, g_{j}\right)\right\}$. Let

$$
A=B \cup C, \quad \text { where } B=\left\{m_{k}: k \in \mathbb{Z}_{+}\right\} \quad \text { and } \quad C=\left\{m_{k}+1: k \in \mathbb{Z}_{+}\right\} .
$$

Clearly $A$ is an infinite countable set. According to (Q4), $\left\|g_{j}\right\| \leqslant 3$ if $j \in B$. By Lemma 2.2 , there exists a bijection $\varphi: \mathbb{Z}_{+} \rightarrow A$ such that $\left\|g_{\varphi(j)}\right\| \leqslant b_{j}$ for each $j \in \mathbb{Z}_{+}$. Now we consider the map $\pi: \mathbb{Z}_{+} \rightarrow \mathbb{Z}_{+}$defined by the formula

$$
\pi(j)= \begin{cases}\varphi(k) & \text { if } j=n_{k}, \\ m_{k}+l+1 & \text { if } j=n_{k}+l, 1 \leqslant l<n_{k+1}-n_{k} .\end{cases}
$$

Using the equalities $m_{k}=n_{k}+k$ and $m_{k+1}-m_{k}=n_{k+1}-n_{k}+1$, it is easy to verify that $\pi$ maps bijectively $\mathbb{Z}_{+} \backslash\left\{n_{k}: k \in \mathbb{Z}_{+}\right\}$onto $\mathbb{Z}_{+} \backslash A$. Since $\varphi: \mathbb{Z}_{+} \rightarrow A$ is also bijective, we have that $\pi: \mathbb{Z}_{+} \rightarrow \mathbb{Z}_{+}$is a bijection. Thus, $\left\{\left(x_{j}, f_{j}\right): j \in \mathbb{Z}_{+}\right\}$ with $\left(x_{j}, f_{j}\right)=\left(y_{\pi(j)}, g_{\pi(j)}\right)$ is just a permutation of the biorthogonal sequence $\left\{\left(y_{j}, g_{j}\right): j \in \mathbb{Z}_{+}\right\}$. Hence $\left\{\left(x_{j}, f_{j}\right): j \in \mathbb{Z}_{+}\right\}$is also a biorthogonal sequence and the properties (B1) and (B2) follow immediately from (Q2) and (Q3). Since $\pi\left(\left\{n_{k}+1, \ldots, n_{k+1}-1\right\}\right)=\left\{m_{k}+2, \ldots, m_{k+1}-1\right\}$ for each $k \in \mathbb{Z}_{+}$, properties (B4) and (B5) follow from (Q4) and (Q5), respectively. Finally $\left\|f_{n_{k}}\right\|=\left\|g_{\varphi(k)}\right\| \leqslant b_{k}$ for each $k \in \mathbb{Z}_{+}$and (B3) is also satisfied.

We need Proposition 2.1 for one purpose only. Namely, we need it in order to prove the following lemma, which is one of the two main ingredients in the proof of Theorem 1.1.

Lemma 2.5. Let $a \in \ell_{2}(\mathbb{N})$ and $T_{a}$ be the weighted backward shift on $\ell_{1}$ with the weight sequence $a$, that is, $T_{a} e_{0}=0$ and $T_{a} e_{n}=a_{n} e_{n-1}$ for $n \geqslant 1$, where $\left\{e_{n}\right\}_{n \in \mathbb{Z}_{+}}$is the canonical basis in $\ell_{1}$. Also let $X$ be a separable infinite dimensional Banach space. Then there exist $T \in L(X)$ and an injective bounded linear operator $J: \ell_{1} \rightarrow X$ with dense range such that $J T_{a}=T J$.

Proof. Since $a \in \ell_{2}(\mathbb{N})$, we can choose a sequence $\left\{n_{k}: k \in \mathbb{Z}_{+}\right\}$of non-negative integers and a sequence $\left\{b_{k}: k \in \mathbb{Z}_{+}\right\}$of numbers in $[3, \infty)$ such that $n_{0}=0$, $n_{k+1}-n_{k} \geqslant 2$ for each $k \in \mathbb{Z}_{+}, b_{k} \rightarrow \infty$ as $k \rightarrow \infty$,

$$
\sum_{k=0}^{\infty} b_{k}\left|a_{n_{k}}\right|<\infty \text { and } \sum_{k=0}^{\infty}\left(\sum_{j=n_{k}+1}^{n_{k+1}}\left|a_{j}\right|^{2}\right)^{1 / 2}<\infty .
$$

Let $\left\{\left(x_{j}, f_{j}\right): j \in \mathbb{Z}_{+}\right\}$be the biorthogonal system in $X \times X^{*}$ furnished by Proposition 2.1 with the above choice of sequences $\left\{n_{k}\right\}$ and $\left\{b_{k}\right\}$. Since $\left\|x_{n}\right\|=1$ for each $n \in \mathbb{Z}_{+}$, the formula

$$
J u=\sum_{n=0}^{\infty} u_{n} x_{n}
$$

defines a bounded linear operator from $\ell_{1}$ to $X$. Since $f_{j}(J u)=u_{j}$ for any $j \in \mathbb{Z}_{+}$ and $u \in \ell_{1}$, we see that $J$ is injective. Since $J\left(e_{j}\right)=x_{j}$ for each $j \in \mathbb{Z}_{+}$and 
span $\left\{x_{j}: j \in \mathbb{Z}_{+}\right\}$is dense in $X$, the operator $J$ has dense range. For each $k \in \mathbb{Z}_{+}$, we consider $S_{k} \in L(X)$ defined by the formula

$$
S_{k} x=\sum_{j=n_{k}+2}^{n_{k+1}-1} a_{j} f_{j}(x) x_{j-1}
$$

assuming that $S_{k}=0$ if $n_{k}-n_{k-1}=2$. Applying Proposition 2.1 (B5) and (B4), we have

$$
\left\|S_{k} x\right\|^{2} \leqslant 4 \sum_{j=n_{k}+2}^{n_{k+1}-1}\left|a_{j}\right|^{2}\left|f_{j}(x)\right|^{2} \leqslant 36\|x\|^{2} \sum_{j=n_{k}+2}^{n_{k+1}-1}\left|a_{j}\right|^{2} \leqslant 36\|x\|^{2} \sum_{j=n_{k}+1}^{n_{k+1}}\left|a_{j}\right|^{2} .
$$

Hence

$$
\left\|S_{k}\right\| \leqslant 6\left(\sum_{j=n_{k}+1}^{n_{k+1}}\left|a_{j}\right|^{2}\right)^{1 / 2}
$$

Thus, applying the second inequality in (2.5), we see that $\sum_{k=0}^{\infty}\left\|S_{k}\right\|<\infty$, and therefore the formula

$$
T_{0} x=\sum_{k=0}^{\infty} S_{k} x
$$

defines a bounded linear operator on $X$. Since by Proposition 2.1 (B3), $\left\|f_{n_{k}}\right\| \leqslant b_{k}$, we, using the first inequality in (2.5), see that the formula

$$
T_{1} x=\sum_{k=1}^{\infty} a_{n_{k}} f_{n_{k}}(x) x_{n_{k}-1}
$$

also defines a bounded linear operator on $X$. Finally, the second inequality in (2.5) implies that $\sum_{k=0}^{\infty}\left|a_{n_{k}+1}\right|<\infty$. Thus, since $\left\|f_{n_{k}+1}\right\| \leqslant 3$ according to Proposition 2.1 (B4), the formula

$$
T_{2} x=\sum_{k=0}^{\infty} a_{n_{k}+1} f_{n_{k}+1}(x) x_{n_{k}}
$$

defines a bounded linear operator on $X$ as well. Consider now the operator $T=$ $T_{0}+T_{1}+T_{2} \in L(X)$. It is straightforward to verify that $T x_{0}=0$ and $T x_{n}=a_{n} x_{n-1}$ for $n \geqslant 1$. Taking into account that $J e_{n}=x_{n}$ for each $n \in \mathbb{Z}_{+}$, we see that $J T_{a} e_{0}=T J e_{0}=0$ and $J T_{a} e_{n}=T J e_{n}=a_{n} x_{n-1}$ for $n \geqslant 1$. Hence $J T_{a}=T J$.

2.2. Orbits of $I-c V$ and $(I+c V)^{-1}$. Let $V$ be the classical Volterra operator acting on $L_{2}[0,1]$ :

$$
V f(t)=\int_{0}^{t} f(s) d s .
$$

We need the following fact on the behavior of the orbits of $I-c V$ and $(I+c V)^{-1}$ for $c>0$. For the case $c=1$, the proof can be found in [18] and the proof for general $c>0$ goes along the same lines. We include the proof for the sake of completeness.

Lemma 2.6. Let $c>0$. Then for any $f \in L_{2}[0,1]$,

$$
\lim _{n \rightarrow \infty}\left\|(I-c V)^{n} f\right\|=0
$$

and

$$
\lim _{n \rightarrow \infty}\left\|(I+c V)^{-n} f\right\|=0
$$


Proof. First, take the function $\mathbf{1}$ being identically 1 . One can easily verify that $(I-c V)^{n} \mathbf{1}(t)=L_{n}(c t)$, where $L_{n}$ are the Laguerre polynomials:

$$
L_{n}(t)=\sum_{k=0}^{n} \frac{n !(-t)^{k}}{(n-k) !(k !)^{2}} .
$$

From the well-known asymptotic formulae [20, Chapter 8] for Laguerre polynomials it follows that for any $0<a<b<\infty,\left\|L_{n}\right\|_{L_{\infty}[0, b]}=O(1)$ and $\left\|L_{n}\right\|_{L_{\infty}[a, b]}=$ $O\left(n^{-1 / 2}\right)$ as $n \rightarrow \infty$. Since $(I-c V)^{n} \mathbf{1}(t)=L_{n}(c t)$, we immediately obtain $(I-c V)^{n} \mathbf{1} \rightarrow 0$ in $L_{2}[0,1]$ as $n \rightarrow \infty$. Now, let $p(t)=\sum_{j=0}^{n} p_{j} t^{j}$ be any polynomial. Then $p=q(V) \mathbf{1}$, where $q(t)=\sum_{j=0}^{n} j ! p_{j} t^{j}$. Therefore

$$
(I-c V)^{n} p=(I-c V)^{n} q(V) \mathbf{1}=q(V)(I-c V)^{n} \mathbf{1} \rightarrow 0 \quad \text { as } n \rightarrow \infty
$$

since $(I-c V)^{n} \mathbf{1} \rightarrow 0$ and $q(V)$ is a bounded linear operator on $L_{2}[0,1]$. Since the space of polynomials is dense in $L_{2}[0,1]$, we see that $(2.6)$ is satisfied for $f$ from a dense set.

Next, $V+V^{*}=P$, where $P$ is the orthoprojection onto the one dimensional space of constant functions. Hence

$$
\operatorname{Re}\langle V f, f\rangle=\frac{1}{2}\left\langle\left(V+V^{*}\right) f, f\right\rangle=\frac{1}{2}\langle P f, f\rangle \geqslant 0 \quad \text { for any } f \in L_{2}[0,1] .
$$

Thus, for any $f \in L_{2}[0,1]$ with $\|f\|=1$, we have

$$
\|(I+c V) f\| \geqslant \operatorname{Re}\langle f+c V f, f\rangle \geqslant\langle f, f\rangle=1 .
$$

Hence $(I+c V)^{-1}$ is a contraction. One can also easily verify that $I-c V=$ $M_{c}(I+c V)^{-1} M_{c}^{-1}$, where $M_{c} f(t)=e^{-c t} f(t)$. It follows that $I-c V$ and $(I+c V)^{-1}$ are similar. Therefore, the operator $I-c V$ is power bounded since it is similar to a contraction. Since $I-c V$ is power bounded and (2.6) is satisfied for $f$ from a dense set, we obtain that (2.6) is satisfied for all $f \in L_{2}[0,1]$. Finally similarity of $I-c V$ and $(I+c V)^{-1}$ implies that (2.7) is also satisfied for any $f \in L_{2}[0,1]$.

2.3. The Kitai criterion and quasisimilarity. It has been noticed by many authors that for any operator satisfying a certain cyclicity property (such as being cyclic, supercyclic, hypercyclic, satisfying the hypercyclicity criterion or satisfying the Kitai criterion), any other operator being in a certain quasisimilarity relation with the first one satisfies the same property. The following lemma is proved in [17].

Lemma 2.7. Let $X$ and $X_{0}$ be Banach spaces, $T_{0} \in L\left(X_{0}\right)$ and $T \in L(X)$. Suppose also that $T_{0}$ satisfies the Kitai criterion and there exists an injective bounded linear operator $J: X_{0} \rightarrow X$ with dense range such that $J T_{0}=T J$. Then $T$ satisfies the Kitai criterion.

\subsection{The Kitai criterion for $I+T$, where $T$ is a backward weighted shift.}

Remark. Let $w=\left\{w_{n}\right\}_{n \in \mathbb{N}}$ and $u=\left\{u_{n}\right\}_{n \in \mathbb{N}}$ be two bounded sequences of nonzero numbers in $\mathbb{K}$ such that $\left|w_{n}\right|=\left|u_{n}\right|$ for each $n \in \mathbb{N}$, and let $T_{w}, T_{u}$ be backward weighted shifts with weight sequences $w$ and $u$ respectively acting on $X$ that are either $\ell_{p}$ for $1 \leqslant p<\infty$ or $c_{0}$. Then the operators $T_{w}$ and $T_{u}$ are isometrically similar. Indeed, consider the sequence $\left\{d_{n}\right\}_{n \in \mathbb{Z}_{+}}$defined as $d_{0}=1$ and $d_{n}=\prod_{j=1}^{n} \frac{w_{j}}{u_{j}}$ for $n \geqslant 1$. Then $\left|d_{n}\right|=1$ for each $n \in \mathbb{Z}_{+}$, and therefore the diagonal operator $D \in L(X)$, which acts on the basis vectors according to the 
formula $D e_{n}=d_{n} e_{n}$ for $n \in \mathbb{Z}_{+}$, is an isometric isomorphism of $X$ onto itself. One can easily verify that $T_{u}=D^{-1} T_{w} D$. That is, $T_{w}$ and $T_{u}$ are isometrically similar.

For a bounded linear operator $S$ on a Banach space $X$ we denote

$$
\mathcal{E}(S)=\left\{x \in X: S^{n} x \rightarrow 0 \text { as } n \rightarrow \infty\right\} .
$$

It follows immediately from Definition 2 that an invertible $S \in L(X)$ satisfies the Kitai criterion if and only if both $\mathcal{E}(S)$ and $\mathcal{E}\left(S^{-1}\right)$ are dense in $X$.

Lemma 2.8. Let $\lambda \in \mathbb{K} \backslash\{0\}$ and let $T$ be the weighted backward shift on $\ell_{1}$ with the weight sequence $w_{n}=1 / n, n \in \mathbb{N}$. Then the operator $I+\lambda T$ satisfies the Kitai criterion.

Proof. Let $c=|\lambda| / 2$. Since $T$ is quasinilpotent, the operator $I+\lambda T$ is invertible and therefore it suffices to prove that $\mathcal{E}(I+\lambda T)$ and $\mathcal{E}\left((I+\lambda T)^{-1}\right)$ are dense in $\ell_{1}$. According to the above remark the operators $\lambda T, 2 c T$ and $-2 c T$ are isometrically similar. Hence $I+\lambda T$ is similar to $I-2 c T$ and $(I+\lambda T)^{-1}$ is similar to $(I+2 c T)^{-1}$. Thus, it suffices to prove that

$$
\mathcal{E}(I-2 c T) \text { is dense in } \ell_{1}
$$

and

$$
\mathcal{E}\left((I+2 c T)^{-1}\right) \text { is dense in } \ell_{1} .
$$

Consider the bounded linear operator $J_{0}: \ell_{\infty} \rightarrow L_{2}[0,1]$ defined by the formula

$$
J_{0} x(t)=\sum_{n=0}^{\infty} \frac{x_{n}(1-t)^{n}}{2^{n}} .
$$

Naturally identifying $\ell_{\infty}$ with $\ell_{1}^{*}$ and $\left(L_{2}[0,1]\right)^{*}$ with $L_{2}[0,1]$, one can easily see that $J_{0}$ is $*$-weakly continuous and therefore $J_{0}=J^{* *}$, where $J=\left.J_{0}\right|_{c_{0}}$. The uniqueness theorem for analytic functions implies that $J_{0}=J^{* *}$ is injective and therefore $J^{*}: L_{2}[0,1] \rightarrow \ell_{1}$ has dense range. Clearly $J\left(c_{0}\right)$ contains all polynomials and therefore is dense in $L_{2}[0,1]$. Hence $J^{*}$ is injective. A direct calculation shows that the dual of the Volterra operator $V$ on $L_{2}[0,1]$ acts according to the formula

$$
V^{*} f(t)=\int_{t}^{1} f(s) d s .
$$

Consider also the weighted forward shift $S \in L\left(c_{0}\right), S e_{n}=e_{n+1} /(n+1)$ for $n \in \mathbb{Z}_{+}$, where $\left\{e_{n}\right\}_{n \in \mathbb{Z}_{+}}$is the canonical basis of $c_{0}$. We shall verify that

$$
2 J S=V^{*} J \text {. }
$$

Indeed, for any $n \in \mathbb{Z}_{+}$,

$$
\begin{aligned}
\left(2 J S e_{n}\right)(t) & =\frac{2\left(J e_{n+1}\right)(t)}{n+1}=\frac{2(1-t)^{n+1}}{(n+1) 2^{n+1}}=\frac{(1-t)^{n+1}}{(n+1) 2^{n}} \\
\text { and } \quad\left(V^{*} J e_{n}\right)(t) & =\int_{t}^{1} \frac{(1-s)^{n}}{2^{n}} d s=\frac{(1-t)^{n+1}}{(n+1) 2^{n}} .
\end{aligned}
$$

Thus, $2 J S e_{n}=V^{*} J e_{n}$ for each $n \in \mathbb{Z}_{+}$and (2.11) follows. Taking the adjoint of both sides of the equality (2.11) and taking into account that $S^{*}=T$, we obtain $2 T J^{*}=J^{*} V$, which immediately implies

$$
(I-2 c T) J^{*}=J^{*}(I-c V) \text { and }(I+2 c T)^{-1} J^{*}=J^{*}(I+c V)^{-1} .
$$


Applying Lemma 2.6, we see that for any $f \in L_{2}[0,1]$,

$$
(I-2 c T)^{n} J^{*} f=J^{*}(I-c V)^{n} f \rightarrow 0
$$

and

$$
(I+2 c T)^{-n} J^{*} f=J^{*}(I+c V)^{-n} f \rightarrow 0 \text { as } n \rightarrow \infty .
$$

Hence each of the spaces $\mathcal{E}(I-2 c T)$ and $\mathcal{E}\left((I+2 c T)^{-1}\right)$ contains $J^{*}\left(L_{2}[0,1]\right)$, which is dense in $\ell_{1}$. Thus, (2.9) and (2.10) hold and therefore $I+\lambda T$ satisfies the Kitai criterion.

2.5. Proof of Theorem 1.2. Condition (1.1) implies that there exists $c>0$ for which the sequence

$$
d_{0}=1, \quad d_{n}=\prod_{j=1}^{n} \frac{c}{n w_{n}} \quad \text { for } n \in \mathbb{N}
$$

is bounded. Let $S$ be the weighted backward shift on $\ell_{1}$ with the weight sequence $w_{n}=1 / n, n \in \mathbb{N},\left\{e_{n}\right\}_{n \in \mathbb{Z}_{+}}$be the canonical basis in $X$ and $\left\{e_{n}^{\prime}\right\}_{n \in \mathbb{Z}_{+}}$be the canonical basis in $\ell_{1}$. Since $\left\{d_{n}\right\}_{n \in \mathbb{Z}_{+}}$is bounded there exists a unique bounded linear operator $J: \ell_{1} \rightarrow X$ such that $J e_{n}^{\prime}=d_{n} e_{n}$ for $n \in \mathbb{Z}_{+}$. Since $d_{n} \neq 0$ for each $n \in \mathbb{Z}_{+}$, the operator $J$ is injective and has dense range. Using the definition of $d_{n}$, one can easily verify that $T J=c J S$ and therefore $(I+T) J=J(I+c S)$. By Lemma 2.8, $I+c S$ satisfies the Kitai criterion. From Lemma 2.7 it follows now that $I+T$ also satisfies the Kitai criterion.

2.6. Proof of Theorem 1.1. Let $S$ be the weighted backward shift on $\ell_{1}$ with the weight sequence $w_{n}=1 / n, n \in \mathbb{N}$. By Lemma 2.5, there exist $T \in L(X)$ and an injective bounded linear operator $J: \ell_{1} \rightarrow X$ with dense range such that $J S=T J$. Hence $J(I+S)=(I+T) J$. By Lemma 2.8, $I+S$ satisfies the Kitai criterion. Now Lemma 2.7 implies that $I+T$ also satisfies the Kitai criterion. The proof is complete.

\section{Concluding Remarks}

The operator that we construct in the proof of Theorem 1.1 is the sum of the identity operator and a compact quasinilpotent operator. In particular, its spectrum is the one-point set $\{1\}$. It is worth noting that there are Banach spaces, where one can not expect anything else. Namely, if $X$ is a hereditarily indecomposable Banach space [3], then the spectrum of any hypercyclic operator $T$ on $X$ is a one-point set $\{\lambda\}$ with $|\lambda|=1$; see, for instance, [13].

The sufficient condition of frequent hypercyclicity [6], an interesting concept recently introduced by Bayart and Grivaux, is related to a stronger form of the Kitai criterion. Let us say that a bounded linear operator $T$ on a Banach space $X$ satisfies the strong Kitai criterion if the spaces $E$ and $F$ in Definition 2 may be chosen to be the same space: $E=F$. Clearly, an invertible operator satisfies the strong Kitai criterion if and only if $\mathcal{E}(T) \cap \mathcal{E}\left(T^{-1}\right)$ is dense in $X$. We shall immediately see that this condition is much more restrictive than the Kitai criterion.

Lemma 3.1. Let $T$ be a bounded linear operator on a complex Banach space $X$ and $\sigma(T)=\{1\}$. Then $\mathcal{E}(T) \cap \mathcal{E}\left(T^{-1}\right)=\{0\}$. 
Proof. Let $Y$ be the space $\mathcal{E}(T) \cap \mathcal{E}\left(T^{-1}\right)$ endowed with the norm $\|y\|_{Y}=$ $\max \left\{\left\|T^{n} y\right\|: n \in \mathbb{Z}\right\}$. It is straightforward to see that $Y$ is a Banach space, $T(Y) \subseteq Y$ and the restriction $A=\left.T\right|_{Y}$, considered as an operator acting on the Banach space $Y$, is an invertible isometry. Now, if $z \in \mathbb{C} \backslash\{1\}$, then, using the fact that $T-I$ is quasinilpotent, we see that $(T-z I)^{-1}=(1-z)^{-1} \sum_{k=0}^{\infty}(1-z)^{k}(T-I)^{k}$, where the series on the right-hand side is operator norm absolutely convergent. Note that if $S$ is a bounded linear operator on $X$ such that $S T=T S$, then $S(Y) \subseteq Y$ and the restriction of $S$ to $Y$, considered as an operator on $Y$, has the operator norm not exceeding $\|S\|$. Hence, the series $(1-z)^{-1} \sum_{k=0}^{\infty}(1-z)^{k}(A-I)^{k}$ is operator norm absolutely convergent to $(A-z I)^{-1}$. Thus, $z \notin \sigma(A)$. Hence, $\sigma(A)=\{1\}$. Since $A$ is an isometry, we have that $\left\{\|A\|^{n}\right\}_{n \in \mathbb{Z}}$ is bounded. A classical theorem due to Gel'fand asserts that an invertible element $x$ of a unital Banach algebra is the identity if $\sigma(x)=\{1\}$ and $\left\{\left\|x^{n}\right\|\right\}_{n \in \mathbb{Z}}$ is bounded. Thus, $A=I$ and therefore $T y=y$ for any $y \in Y$. From the definition of $Y$ it follows that $T y=y$ for $y \in Y$ happens if and only if $y=0$. Thus, $Y=\{0\}$.

Proposition 3.2. Let $X$ be a hereditarily indecomposable Banach space. Then there is no bounded linear operators on $X$ satisfying the strong Kitai criterion.

Proof. The case $\mathbb{K}=\mathbb{R}$ reduces to the case $\mathbb{K}=\mathbb{C}$ by passing to the complexification. Thus, we can assume that $\mathbb{K}=\mathbb{C}$. Let $S$ be a bounded linear operator on $X$, satisfying the strong Kitai criterion. As we have already mentioned, the fact that $X$ is hereditarily indecomposable implies that $\sigma(S)$ is a one-point set $\{\lambda\}$ with $|\lambda|=1$. Then $T=\lambda^{-1} S$ satisfies the strong Kitai criterion and $\sigma(T)=\{1\}$. Since $T$ is invertible and satisfies the strong Kitai criterion, we have that $\mathcal{E}(T) \cap \mathcal{E}\left(T^{-1}\right)$ is dense in $X$, which is not possible according to Lemma 3.1.

It is also worth noting that Theorem 1.2 is surprisingly sharp. The following observation is due to Atzmon; see [4, 5].

Theorem A. Let $k \in \mathbb{N}$ and let $T$ be a bounded linear operator on a Banach space $X$ such that $\left\|T^{n}\right\|^{1 / n}=o(1 / n)$ as $n \rightarrow \infty$. Then for $x \in X,\left\|(I+T)^{n} x\right\|=O\left(n^{k}\right)$ as $n \rightarrow \infty$ if and only if $T^{k} x=0$.

From Theorem A it immediately follows that $\mathcal{E}(I+T)=\{0\}$ if $\left\|T^{n}\right\|^{1 / n}=o(1 / n)$ as $n \rightarrow \infty$. Indeed, if $x \in \mathcal{E}(I+T)$, then by Theorem $\mathrm{A}$ we have $T x=0$ and therefore $(I+T)^{n} x=x$ for each $n \in \mathbb{Z}_{+}$. Taking into account that $\mathcal{E}(S)$ is dense for each operator $S$ satisfying the Kitai criterion, we have the following corollary.

Corollary 3.3. Let $T$ be a bounded linear operator on a Banach space $X$ such that $\left\|T^{n}\right\|^{1 / n}=o(1 / n)$ as $n \rightarrow \infty$. Then $I+T$ does not satisfy the Kitai criterion.

From Corollary 3.3 it follows, in particular, that if $T$ is a weighted backward shift on $\ell_{p}$ for $1 \leqslant p<\infty$ or on $c_{0}$ with the weight sequence $\left\{w_{n}\right\}$ satisfying $w_{n}=o\left(n^{-1}\right)$ as $n \rightarrow \infty$, then $I+T$ does not satisfy the Kitai criterion.

It is well known and easy to see that the spectrum of a backward weighted shift $T$ is always the disk $\{z \in \mathbb{C}:|z| \leqslant r\}$, where $r \geqslant 0$ is the spectral radius of $T$. As easily follows from the results of Chan and Shapiro [9], $I+T$ satisfies the Kitai criterion if $r>0$. From this point of view Theorem 1.2 gives a much more subtle sufficient condition for such operators to satisfy the Kitai criterion. In particular, it shows that there are many quasinilpotent backward weighted shifts $T$ for which $I+T$ satisfies the Kitai criterion. 
Finally observe that the Kitai criterion makes sense for operators on arbitrary topological vector spaces. A natural question arises in relation with Theorem 1.1. Namely, does there exist a continuous linear operator satisfying the Kitai criterion on any separable infinite dimensional Fréchet space?

\section{ACKNOWLEDGMENTS}

The author is grateful to Professors Sophie Grivaux and Charles Read for their interest and helpful comments.

\section{REFERENCES}

[1] S. Ansari, Hypercyclic and cyclic vectors, J. Funct. Anal. 128 (1995), 374-383 MR1319961 (96h:47002)

[2] S. Ansari, Existence of hypercyclic operators on topological vector spaces, J. Funct. Anal. 148 (1997), 384-390 MR1469346 (98h:47028a)

[3] S. Argyros and A. Tolias, Methods in the theory of hereditarily indecomposable Banach spaces, Mem. Amer. Math. Soc. 170 (2004), 1-114 MR2053392 (2005f:46022)

[4] A. Atzmon, Operators which are annihilated by analytic functions and invariant subspaces, Acta Math. 144 (1980), 27-63 MR558090 (81c:47007)

[5] A. Atzmon, On the existence of invariant subspaces, J. Operator Theory 11 (1984), 3-40 MR739792 (85k:47005)

[6] F. Bayart and S. Grivaux, Frequently hypercyclic operators, Trans. Amer. Math. Soc. 358 (2006), 5083-5117 MR2231886 (2007e:47013)

[7] L. Bernal-González, On hypercyclic operators on Banach spaces, Proc. Amer. Math. Soc. 127 (1999), 1003-1010 MR1476119 (99f:47010)

[8] J. Bès and A. Peris, Hereditarily hypercyclic operators, J. Funct. Anal. 167 (1999), 94-112 MR1710637 (2000f:47012)

[9] K. Chan and J. Shapiro, The cyclic behavior of translation operators on Hilbert spaces of entire functions, Indiana Univ. Math. J. 40 (1991), 1421-1449 MR1142722 (92m:47060)

[10] G. Costakis and M. Sambarino, Topologically mixing operators, Proc. Amer. Math. Soc. 132 (2003), 385-389 MR2022360 (2004i:47017)

[11] A. Dvoretzky, A theorem on convex bodies and application to Banach spaces, Proc. Nat. Acad. Sci. USA 45 (1959), 223-226 MR0105652 (21:4391)

[12] G. Herzog, On linear operators having supercyclic vectors, Studia Math. 103 (1992), 295-298 MR1202014 (93k:47033)

[13] S. Grivaux, Hypercyclic operators, mixing operators and the bounded steps problem, J. Operator Theory 54 (2005), 147-168 MR2168865 (2006k:47021)

[14] K. Grosse-Erdmann, Universal families and hypercyclic operators, Bull. Amer. Math. Soc. 36 (1999), 345-381 MR1685272 (2000c:47001)

[15] K. Grosse-Erdmann, Recent developments in hypercyclicity, RACSAM Rev. R. Acad. Cienc. Exactas Fis. Nat. Ser. A Mat. 97 (2003), 273-286 MR2068180 (2005c:47010)

[16] C. Kitai, Invariant closed sets for linear operators, Thesis, University of Toronto, 1982

[17] F. Martínez-Giménez and A. Peris, Chaos for backward shift operators, Internat. J. Bifur. Chaos Appl. Sci. Engrg. 12 (2002), 1703-1715 MR1927407 (2003h:47056)

[18] A. Montes-Rodríguez, J. Sánchez-Álvarez and J. Zemánek, Uniform Abel-Kreiss boundedness and the extremal behaviour of the Volterra operator, Proc. London Math. Soc. 91 (2005), 761-788 MR2180462 (2006j:47053)

[19] H. Salas, Hypercyclic weighted shifts, Trans. Amer. Math. Soc. 347 (1995), 993-1004 MR1249890 (95e:47042)

[20] G. Szegö, Orthogonal polynomials, Amer. Math. Soc., Colloq. Publ., vol. 23, 1959 MR0106295 $(21: 5029)$

Department of Pure Mathematics, Queen's University Belfast, University Road, BT7 1NN Belfast, United Kingdom

E-mail address: s.shkarin@qub.ac.uk 